\title{
Correction to: Risk of adverse outcomes in patients with rheumatoid arthritis hospitalized for stroke-a cross-sectional study
}

\author{
Jiunn-Horng Kang ${ }^{1,2} \cdot$ Sudha Xirasagar ${ }^{3} \cdot$ Herng-Ching Lin $^{4,5}$ (D) $\cdot$ Pai-Feng Kao ${ }^{6,7} \cdot$ Li-Chin Sung $^{6,7}$ \\ Published online: 6 April 2019 \\ (C) International League of Associations for Rheumatology (ILAR) 2019
}

Correction to: Clinical Rheumatology (2018) 37:2917-2926

https://doi.org/10.1007/s10067-018-4287-8

The authors of the published version of this article incorrectly presented the affiliation of Li-Chin Sung, this should have read, "Division of Cardiology, Department of Internal Medicine, School of Medicine, College of Medicine, Taipei Medical University, Taipei, Taiwan" The revised affiliation is now presented correctly in this article.

The remainder of the article remains unchanged.

Publisher's note Springer Nature remains neutral with regard to jurisdictional claims in published maps and institutional affiliations.

The online version of the original article can be found at https:/doi.org/ $10.1007 / \mathrm{s} 10067-018-4287-8$

Herng-Ching Lin

henry11111@tmu.edu.tw

1 Department of Physical Medicine and Rehabilitation, Taipei Medical University Hospital, Taipei, Taiwan

2 Department of Physical Medicine and Rehabilitation, School of Medicine, College of Medicine, Taipei Medical University, Taipei, Taiwan

3 Department of Health Services Policy and Management, Arnold School of Public Health, University of South Carolina, Columbia, SC, USA

4 School of Health Care Administration, Taipei Medical University, $250 \mathrm{Wu}-H s i n g$ St, Taipei 110, Taiwan

5 Sleep Research Center, Taipei Medical University Hospital, Taipei, Taiwan

6 Division of Cardiology, Department of Internal Medicine, School of Medicine, College of Medicine, Taipei Medical University,

Taipei, Taiwan

7 Division of Cardiology, Department of Internal Medicine, Shuang Ho Hospital, Taipei Medical University Taipei, Taipei, Taiwan 